\title{
Simulation Evaluation Algorithms of LSRP and DVRP in Bank Network
}

\author{
Feixue Huang \\ School of Management, Dalian University of Technology, Dalian 116024, China \\ Tel: 86-411-8470-7210Ｅ-mail: software666@163.com \\ Yong Zhou \\ Dalian Branch, Bank of Communications, Co., Ltd, Dalian 116001, China \\ Tel: 86-411-86-8281-6791Ｅ-mail: unite@sina.com
}

Zhijie Li

School of Computer Science \& Engineering, Dalian Nationalities University, Dalian 116600, China

Tel: 86-411-8765-6179 E-mail: lizhijie@dlnu.edu.cn

\begin{abstract}
Because the computational complexity of high usability analysis is too high when selecting the routing protocol in the bank network plan, a simple measurement method of high usability for banks is proposed to solve this problem in the article. First, establish a simulation environment which is close to the real network of banks to offer the measurement environment and data. Second, based on the theoretical comparison of LSRP and DVRP, establish the simple simulation measurement algorithm by Shannon's information entropy theory. Finally, evaluate the degree of high usability of LSRP and DVRP in the bank network by this algorithm. The result of simulation shows that (1) the simulation is close to the periodic rule of the statistical data group in real environment, (2) the deviation ratio is less than 0.1 , and (3) the covariance is unequal to 0 . And the result indicates that the simulation is connected with the real environment and both are very close, and the simulation environment can offer effective data. The computation result of the simple simulation measurement algorithm shows that the time cost differences of LSRP and DVRP exist in the period of fault recovering, which indicates the simple measurement algorithm is effective.
\end{abstract}

Keywords: Network system, Distance vector routing protocol (DVRP), Link-state routing protocol (LSRP), High usability, Information entropy

\section{Introduction}

Up to the late of 2008, the development of network payment associating with network shopping develops quickly, and the using scale has achieved 52 million people at present, and the yearly increase rate achieves $57.6 \%$. The people who use the web bank have achieved to 58 millions in 2008 from 40 millions in 2007, and the increase rate achieves $45 \%$ (China Internet Network Information Center, 2009). By the scientific and technological measures, various commercial banks create financial products actively, and the IT investment scale of Chinese bank industry in 2005 had achieved 23.82 billion Yuan. Up to now, the operation and management systems including office of various commercial banks have all entered into the times of network.

Facing the network faults which can not be avoided completely, people try to enhance the usability of network. The quantitative evaluation method of the usability can respectively define the measurement index based on network connection and the measurement index based on network communication ability (Zhang, 2004, P.103-105). The network usability includes four aspects including equipment, route, user, and application (XF Wang, 2002, P.521-531). Based on the measurement, people put forward the design approaches and design ideas of usable network (C Song, 2005, P.392-395). Aiming at two kinds of damages faced by the complex network, some scholars respectively defined the fault tolerance and the invulnerability for the network node and the network border (Wu, 2005, P.128-131). Some scholars defined the ratio of the possible route amount among network groups to the route amount which could be required to establish among network groups as the usability of network topology (Zhong, 2003, P.113-114). Some scholars also advised seeking the usability values of various nodes in the communication network and taking the mean square deviation of these values as the usability value of the whole network topology (Chen, 2006, P.6-7 \& 24). 
For the network with many nodes, the practice (Lin, 2008, P.15-16) or the space computational complexity (Li, 2008, P.311-318) of general algorithms is too high, so researchers begun to design a more effective algorithm with low computational complexity starting from other approaches, and the concrete ideas could be divided into following sorts. In decomposition method, Kennington and Nair designed the usability network by splitting the network into small loop networks (J L Kennington, 1999, P.219-230), and Kerivin put forward the splitting-solving algorithm based on a series of inequalities (H Kerivin, 2002, P.265-268). Ghashghai and Rardin designed a mixed gene algorithm to split the network into many networks including many trees (E Ghashghai, 2002, P.53-66). Borne and Gourdin et al established a multi-layer usability network design model and put forward the branch cutting algorithm based on the model (S Borne, 2006, P.41-73). In the increase limitation method, Jothi and Raghavachari put forward the minimum generation network problem solving algorithm containing the capability limitation (R Jothi, 2004, P.183-190). Some scholars put forward the algorithm to establish the protection chain (C Liu, 2006, P.301-311), or increase the transportation flux and the route bandwidth limitation in the network (P Broström, 2006, P.235-253) when solving the problem of usability network design, or the comprehensive method (S Soni, 2002, P.133-149). And many other researchers took the routing selection network and the confirmation of the communication chain capacity as the optimized objectives, and used the genetic algorithm (Yue, 2009, P.205-206 \& 209) to solve this complex nonlinear plan problem with many restriction conditions (T. Lux, 2005, P.169-196).

By the research about the computer network usability (Oleszkiewicz J, 2006, P.66-77), three methods respectively had their advantages and disadvantages. The first method studied the bottom theory algorithm and proved that the routing protocol had higher usability than another protocol. This kind of proof is lack of events, which might be because the differences of the data types and the equipment state made the practice result to be different with the result of theoretical analysis. The second method used existing graph theory combining with network factors to compute new algorithm of usability measurement. And various measurement methods in this type should consider numerous nodes, and the measurement algorithm was too complex in actual application. The third method utilizes exiting algorithms and technologies to enhance the usability of existing network, which lacks in the researches and improvement about the used algorithms and technologies.

People try to maintain the usability by the excellent design before the network is established and the good operation after the network is established. In the initial term when the network is established, one or one set of routing protocol fitting for future network should be selected, and this method needs theoretical base and low computational complexity. Therefore, the simulation network can be used to statically compare the running of the usable routing, and establish a measurement algorithm with low computational complexity. First, the simulated network environment of commercial bank is established, and the established environment adopts the mainstream network topology structure, i.e. the star configuration, in Chinese commercial banks, and the connection mode adopts the mainstream double-chain and double-star crossing. And the variance and covariance method is used to validate the close relationship between the simulation environment and the real environment. Second, based on theoretical analysis, the time costs of maintainability generated by three factors including CPU processing, routing convergence and bandwidth occupation are selected (Samuel C.A. Pereira, 2008, P.321-325) when the network fails, and combining with Shannon's theory of information entropy (C.E. Shannon, 1948, P. 379-423 \& 623-656 \& Gianpietro Malescio, 2006, P.918-918) and the randomicity (Liu, 2007, P.350-351), the simple simulation measurement algorithm (with low computational complexity) could be established. By the comparison and analysis of simulation test data, the established simple simulation measurement algorithm can be used to confirm the routing protocol with low time costs is the routing protocol with high usability.

In the article, by the statistical data offered by the established simulation environment, the evaluation measurement algorithm and the theory base are established to select the routing protocol for the large-scale network construction and update alternation in certain bank.

\section{Theoretical comparison of LSRP and DVRP}

The dynamic routing means the router can automatically establish its own routing list and properly adjust it according to the change of actual situation. The operation of the dynamic routing selection algorithm depends on two basic functions, i.e. the maintenance of the routing list, and the proper routing information exchange among routers. The dynamic routing protocol mainly includes the link state routing protocol (LSRP) and the distance vector routing protocol (DVRP).

\subsection{LSRP}

LSRP is the inter-domain routing protocol which is used most widely, and it adopts the design strategy of "jigsaw puzzle", i.e. each router broadcasts its link states with neighbor routers to all other routers in the network. In this way, after one router accepts the routing information sent from other routers, it assembles these link states and generates a topology view of the whole network, so it can compute the shortest route to other routers by the shortest route algorithm. 
The initial LSRP develops from multiple research results, and Dr. Radiap Perlman researching about the information fault tolerance broadcast was the early presenter. At the same time, IETF (Internet Engineering Task Force) issued the algorithm based on the link state in RFC1247, i.e. the OSPF (the routing selection protocol based on the SPF algorithm) (Yan, 2002, P.460-464). The OSPF message refreshing mechanism will generate many messages increasing the load of network, and Chen Zhibo and Xu Mingwei put forward the mechanism of ERR (enhanced randomized refresh). The method which randomly decentralizes the refreshing time of OSPF message and prolongs the refreshing interval of OSPF message can eliminate the abrupt flux brought by OSPF and reduce the amount of OSPF message (Chen, 2005, P.966-968). To control the amount of LSA when refreshing OSPF, Liu Kuixing and Wang Binqiang put forward the random refreshing mechanism of flux control. And this mechanism adopted many technologies such as the slow start and acceleration degression to adjust the amount of LSA and avoid the congestion according to the network flux (Liu, 2007, P.241-242 \& 249).

\subsection{DVRP}

DVRP is based on R. E. Bellman, L. R. Ford and D. R. Fulkerson's works, so it is also called as Bellman-Ford algorithm or the Ford-Fulkerson algorithm.

In DVRP, each router maintains one sheet of routing vector list which lists known best distance (metric) and path to each destination. It refreshes the information in the list by exchanging information with the neighbor routers. DVRP is the route selection algorithm based on Bellman-Ford algorithm.

The core idea of this algorithm is to continually look for the extended routes in the map by the method of tab until the extended routes can not be found. The distance vector routing algorithm requires each router sends its whole routing list when refreshing each times only to its neighbors. The representative application protocols include RIP, IGRP, RTMP and so on.

This algorithm has three deficiencies, i.e. it can not reflect the deterioration, the routing exchange, and the infinite computation. Aiming at these deficiencies, Ding Qin and Cai Yuecai used the split horizon method (Ding, 2008, P.34-36) to improve the running performance of the routing protocol.

\subsection{Comparison of LSRP and DVRP}

Comparing with DVRP, LSRP has quicker convergence speed and higher flexibility, and it can send the link state information to neighbor routers only when the network changes, and store the topology of the whole network and observe the network from its own view. LSRP is a more complex routing protocol than DVRP.

\section{Simulation and measurement algorithm}

Except for prolonging the MTTF of the system, the method to enhance the network usability is to reduce the maintenance time of the system. Next, the problem which one of LSRP and DVRP can enhance the network usability by reducing the maintenance time of the system in the future network of banks will be discussed.

\subsection{Simulation intension and environmrnt}

The intension of the experiment is to establish the simulation environment which is equal to or close to the real environment, and by the analysis and comparison of the statistical data of the real network environment of certain bank with the data collected in the experimental environment, to validate whether the established simulation environment is close to the real environment.

For the experimental environment, the network with 20 nodes is selected to simulate the real network. The network topology adopts the double-star configuration, and the connections between each node and the core in the double-star configuration respectively adopts two $10 / 100 \mathrm{M}$ Ethernets, and two two-layer exchangers are used to simulate the local end of the telecom operator at the connections between the router and every nodes in the core route (seen in Figure 1). Operate the routing protocols including the OSPF (the representative routing selection protocol of LSRP) and the RIP (the representative routing selection protocol of LSRP) in the simulation environment successively. In the time period of 220 seconds, the experiment will collect the statistical data and analyze the time quantities respectively consumed by CPU, convergence and bandwidth.

\subsection{Result and analysis of simulation test}

Figure 2 reflects the statistical comparison of the CUP time consumptions of DVRP and LSRP. LSRP transmits the routing information to neighbor routers per 10 seconds to keep the consistence of the routing list. In the initiatory 63 seconds, CPU works continually to rebuild the neighbor relationship and the synchronous information. DVRP transmits relative routing information per 101 seconds to keep the information synchronization among routers. In the initiatory 25 seconds, CPU works continually to process the routing protocol and synchronous information. The cause to generate this result is that the computation of DVRP is less in the initial term of intermission, and the information of the routing 
list is relatively simple, so the use of CPU is less than the use of LSRP no matter in the convergence process or in receiving data.

Figure 3 reflects the statistical comparison of the convergence time occupations of DVRP and LSRP. LSRP completes the convergence task in 25 seconds, and DVRP needs 63 seconds for the same task. The cause is that both DVRP and LSRP need using their own modes to complete the convergence and occupies the time in this process in the initial term that the network fails. When the convergence completes, DVRP and LSRP will enter into the stable state and won't occupy time because of the convergence process. Because DVRP only transmits the routing list, and the network scale of the experiment is appropriate, and the LSA information transmitted by LSRP is so complex that it needs computation of CPU, so in the experimental environment, the convergence time of LSRP is longer than the convergence time of DVRP.

Figure 4 reflects the statistical comparison of bandwidth times of DVRP and LSRP. The DVRP occupies wider bandwidth than LSRP. The cause to generate this result is that the routing list information synchronization can be completed per 100 seconds. But LSRP only synchronizes the changed information in the period, and if the information doesn't change, LSRP will directly confirm the survival of neighbors. If the data quantity is less, the bandwidth occupation time is less, so DVRP occupies less time than DVRP.

For the statistical analysis of the simulation and reality of LSRP and DVRP, there are two differences between the actual network environment and the simulation environment. The first one is that the network scales are different, and the scale of the real network is 2.5 times than the scale of the simulation network (the amount of network node increases from 20 to 53). The second one is that the network bandwidths are different. The wide area network connection in real network adopts the bandwidth line of $2 \mathrm{M}$ of telecom operator, and it is smaller than the Ethernet of $10 / 100 \mathrm{M}$ in the simulation network.

Figure 5 reflects the statistical comparison between the LSRP simulation and the actual network. The rules that two networks run LSRP are close, but not consistent completely. The cause to generate this result is that though the scale of the actual network is bigger than the scale of the simulation network, the networking equipment and the line bandwidth adopted whether in the simulation environment or in the real environment can completely fulfill the requirements of the network construction of this scale, so even the network scale increases, the change of comprehensive time costs is not obvious.

Figure 6 reflects the measurement results of the DVRP simulation and the actual network. The rules that two networks run DVRP are close, but not consistent completely. The cause to generate this result is that though the scale of the actual network is bigger than the scale of the simulation network, but the networking equipment and the line bandwidth adopted whether in the simulation environment or in the real environment can completely fulfill the requirements of the network construction of this scale, so even the network scale increases, the change of comprehensive time costs is not obvious.

From Figure 5 and Figure 6, the change tendencies of the data of the experimental simulation and the statistical data in the real environment are close which can be reflected in the chart of statistical data. And it can also be proved from exiting algorithm. Next, respectively utilize the variance method and the covariance method to analyze the deviation degree and the association degree of two groups of data from the quantitative angle.

The variance is the value to reflect the centralized degree of data, and it describes the discrete degree of data. For the group data of $\left(x_{1}, x_{2}, \cdots, x_{n}\right)$, its average value is $\bar{x}$, so its variance is

$S^{2}=\frac{1}{n}\left[\left(x_{1}^{2}+x_{2}^{2}+\ldots \ldots+x_{n}^{2}\right)-n \bar{x}^{2}\right]$

Supposed that the variances of the simulation data and the real data respectively are $S_{s}^{2}$ and $S_{r}^{2}$, and the deviation degree is $S_{\Delta}^{2}$, and the deviation rate is $\rho$, so

$S_{\Delta}^{2}=S_{s}^{2}-S_{r}^{2}$

$\rho=S_{\Delta}^{2} \div S_{r}^{2}$

From Table 1, the deviation degree between the data obtained in the simulation experiment of LSRP and the experimental data in the reality is only 0.033716 , and the deviation degree between the data obtained in the simulation experiment of DVRP and the experimental data in the reality is only 0.004238 .

The covariance is to describe the association degree of the data. $\mathrm{X}$ and $\mathrm{Y}$ are two variables, and the covariance can be denoted as

$\operatorname{Cov}(X, Y)=E[(X-E(X))(Y-E(Y))]$ 
If two random variables $\mathrm{X}$ and $\mathrm{Y}$ are independent to each other, so $\operatorname{Cov}(\mathrm{X}, \mathrm{Y})=0$, and if the mathematical anticipation is not zero, so $\mathrm{X}$ and $\mathrm{Y}$ are not independent to each other, i.e. they have certain relationship.

By the computation, the comparison analysis results about LSRP and DVRP can be obtained.

$$
\begin{aligned}
& \operatorname{Cov}_{\text {LSRP }}(X, Y)=3450.373 \neq 0 \\
& \operatorname{Cov}_{\text {DVRP }}(X, Y)=3970.117 \neq 0
\end{aligned}
$$

The result by the method of variance indicates that the deviation degrees between the simulation data and the statistical data are all less than 1 and even 0.1 . Thus deviation degree shows that both are very close. The result computed by the method of covariance is not equal to zero. According to the definition of the covariance, two groups of data are not independent, and have certain associated relationship. By the statistics and computation, the result shows that the established simulation environment can replace the real environment completely.

\subsection{Measurement algorithm}

Except for the factors such as the hardware redundancy, the line redundancy, and the network structure, the factor to enhance the usability of network is the recovering time of network fault. So respectively in LSRP and DVRP the time costs of CPU, convergence, and bandwidth when network fails are compared. The routing protocol with the lowest time costs in above three factors (CPU, convergence, and bandwidth) more fits for the requirements of the future network plan.

Combining with the research result of the information entropy and the complexity degree, the relationship among these three factors is seen in the equation (5).

$T_{c}=T_{c C P U} \times \mathrm{C}_{\mathrm{CPU}}+T_{c C} \times \mathrm{C}_{C}+\mathrm{T}_{\mathrm{c} B} \times \mathrm{C}_{B}$

Where, $T_{c}$ is the total time cost, $T_{c C P U} T_{c C}$ and $T_{c B}$ respectively denote the time costs of CPU, convergence, and bandwidth, and $\mathrm{C}$ can be defined as the equation (6).

$C=-\sum_{i=1}^{k} n_{i} \log \left(\frac{n_{i}}{N}\right)$

Where, $\mathrm{N}$ is the sum of $n_{i}(1 \leq i \leq k)$ numbers, and the probability that any one number occurs is $\frac{n_{i}}{N}$.

The equation (6) is the expression of the information entropy, and it denotes the uncertainty of the random data occurring in the data group.

\subsection{Application of measurement algorithm}

The intention of the measurement algorithm is to apply it into the engineering practice, i.e. the simulation experiment.

Use the equation (5) to compute the time costs of LSRP and DVRP in the simulation environment, and the result is seen in Table 2.

Table 2 lists the time costs of LSRP and DVRP in every time period.

In the initial time periods of 1-40, 41-80, 81-120, and 121-160, the time costs of LSRP are higher than the time costs of DVRP, because the computational complexity of LSRP is higher than that of DVRP, so LSRP will generate higher time costs than DVRP in large-scale network.

In the late time periods of 161-200 and 201-220, the time costs generated by DVRP are higher than LSRP, because after LSRP completes the convergence, it will not occupy the bandwidth of network if the routing changes little.

In the whole time period of 1-220, the time costs generated by DVRP are higher than LSRP, because LSRP sends less information quantity, and it will occupy less bandwidth in large-scale network.

Table 3 clearly lists the analysis results of evaluation of LSRP and DVRP and the causes to generate the result.

Through the analysis, LSRP more suits for the simulated bank network than DVRP in the simulated network structure.

\section{Conclusions}

Because the bank network plan lacks simple simulation method when selecting the routing protocol, the theoretical characters of the dynamic routing protocol are further studied based on former scholars' researches in the article. The measurement model taking the time costs of CPU, convergence, and bandwidth in the fault period by the method of information entropy, i.e. the equation (5), is established. By the experimental data in the simulation environment, the usability of dynamic routing protocol is evaluated. 
First, the method of variance and the method of covariance indicate that the experimental environment established in the article can be realized. Second, the simple algorithm in the article is applied in the simulation data and the evaluation result is obtained. Above results all show that the application of the simulation environment established in the article and the simple measurement algorithm in the concrete engineering practices of large-scale network construction and updating alteration for commercial banks is feasible.

\section{References}

C.E. Shannon. (1948). A mathematical theory of communication. Bell System Technical Journal. No.27. P. $379-423$ \& 623-656.

Chen, Jianguo \& Zhang, Yongjing. (2006). Study on Evaluation Algorithm for Topology Survivability of Communication Network. Radio Communications Technology. No. 32(1). P.6-7 \& 24.

Chen, Zhibo, Xu, Mingwei \& Cui, Yong. (2005). Improved OSPF Refresh Mechanism. Journal of Tsinghua University (Science and Technology). No.45 (7). P.966-968.

China Internet Network Information Center. (2009). The 23 rd Statistical Report on the Internet Development in China. Beijing: China Internet Network Information Center.

C Liu, L Ruan. (2006). P-Cycle design in survivable WDM networks with shared risk link groups (SRLGs). Photonic Network Communications. No.11(3). P.301-311.

C Song, S Havlin \& HA Makse. (2005). Self-similarity of complex networks. Nature. No. 433(7024). P.392-395.

Ding, Qin \& Cai, Yuecai. (2008). Improvement Scheme of Distance Vector Routing Algorithm. Journal of Sichuan University of Science \& Engineering (Natural Science Edition). No.21 (3). P.34-36.

E Ghashghai, R Rardin. (2002). Using ahybrid of exact and genetic algorithms to design survivable networks. Computers \& Operation Research. No. 29. P.53-66.

Gianpietro Malescio. (2006). Coping with uncertainty. Nature. No.443 (7114). P.918-918.

H Kerivin, A R Mahjoub. (2002). Separation of partition inequalities for the (1, 2)-survivable network design problem. Operations Research letters. No.30 (4). P.265-268.

J L Kennington, VSS Nair, M H Rahman. (1999). Optimization Based Algorithms for Finding Minimal Cost Ring Covers in Survivable Networks. Computational Optimization and Applications. No.14 (2). P.219-230.

Li, Zhimin, Luo, Libo \& Li, Xiang. (2008). Study on the Computational Complexity of the Complete Binary Tree. Acta Mathematica Sinica (Chinese Series). No. 51(2). P.311-318.

Lin, Xiaozhu, Wu, Yijuan \& Fu, Dandan. (2008). Analysis of Computation Complexity on Euler Number of a Binary Image. Microelectronics \& Computer. No. 25(7). P.15-16.

Liu, Haoguang \& Cai, Shaohong. (2007). Message Entropy and Randomicity. Journal of Guizhou University (Natural Sciences). No.27(4). P.350-351.

Liu, Kuixing, Wang, Binqiang \& Zhang, Jianhui. (2007). OSPF-LSA Refresh Mechanism with Traffic Control Function. Application Research of Computers. No.24 (9). P.241-242 \& 249.

Oleszkiewicz J, Li X, Liu Y H. (2006). Effectively Utilizing Global Cluster Memory for Large Data-Intensive Parallel Programs. IEEE Transactions on Parallel and Distributed Systems. No.17 (1). P.66-77.

P Broström, K Holmberg. (2006). Multiobjective design of survivable IP networks. Annals of Operations Research. No. 147(1). P.235-253.

R Jothi, B Raghavachari. (2004). Survivable network design: the capacitated minimum spanning network problem. Information Processing Letters. No. 91(4). P.183-190.

Samuel C.A. Pereira, Paulo S.A. Sousa. (2008). Uncertainty and information accuracy in adverse selection. Economics Letters. No.100 (3). P.321-325.

S Borne, E Gourdin, B Liau, et al. (2006). Design of Survivable IP-over-optical networks. Annals of Operations Research. No. 146(1). P.41-73.

S Soni, H Pirkul. (2002). Design of Survivable Networks with Connectivity Requirements. Telecommunication Systems. No. 20(1-2). P.133-149.

T. Lux \& S. Schornstein. (2005). Genetic learning as an explanation of stylized facts of foreign exchange markets. Journal of Mathematical Economics. No.41 (1-2). P.169-196.

Wu, Jun \& Tan, Yuejin. (2005). Study on Measure of Complex Network Invulnerability. Journal of Systems Engineering. No. 20(2). P.128-131. 
XF Wang \& G Chen. (2002). Pinning control of scale-free dynamical networks. Physica A: Statistical Mechanics and its Applications. No. 310(3-4). P.521-531.

Yan, Binyu, Lu, Wei \& Huang, Rui. (2002). A Study on OSPF Routing Protocol. Journal of Sichuan University (Natural Science Edition). No.39 (3). P.460-464.

Yue, Chengjun, Yin, Fengjie \& Jing, Yuanwei. (2009). Multicast Routing Based on Information Entropy Multi-population Genetic Algorithm. Computer Engineering. No.35 (11). P.205-206 \& 209.

Zhang, Yu \& Yang, Xiaojing. (2004). Research on the Simulation Method of Local Communication Network in Field Zone. Computer Engineering. No. 30(8). P.103-105.

Zhong, Lianjiong \& Xu, Feng. (2003). The Computer Network Algorithm of Invulnerability. Fire Control \& Command Control. No. 28(z1). P.113-114.

Table 1. Deviation rates of LSRP and DVRP

\begin{tabular}{|c|c|c|c|c|}
\hline & $\begin{array}{c}\text { Variance of } \\
\text { simulation data }\end{array}$ & $\begin{array}{c}\text { Variance of reality } \\
\text { data }\end{array}$ & $\begin{array}{c}\text { Deviation } \\
\text { degree }\end{array}$ & Deviation rate \\
\hline LSRP & 3407.471 & 3526.366 & 118.8948 & 0.033716 \\
\hline DVRP & 3979.841 & 3996.7803 & 16.93931 & 0.004238 \\
\hline
\end{tabular}

Table 2. Measurement summary of LSRP and DVRP

\begin{tabular}{|c|c|c|}
\hline Time group & $T c_{\text {LSRP }}$ & $T c_{\text {DVRP }}$ \\
\hline $1-40$ & 141483.3020 & 126945.2688 \\
\hline $41-80$ & 778866.0793 & 657273.7579 \\
\hline $81-120$ & 1584038.8700 & 1562649.7680 \\
\hline $121-160$ & 2694021.8310 & 2219259.6360 \\
\hline $161-200$ & 4136163.0950 & 4615592.7640 \\
\hline $201-220$ & 2112812.5600 & 2385727.9990 \\
\hline $1-220$ & 22189385.4300 & 24120264.1900 \\
\hline
\end{tabular}

Table 3. Comparison and analysis of measurement results

\begin{tabular}{|c|c|c|c|}
\hline $\begin{array}{c}\text { Time } \\
\text { period }\end{array}$ & \multicolumn{1}{|c|}{$1-160$} & \multicolumn{1}{|c|}{$161-220$} & $1-220$ \\
\hline LSRP & High time costs & \multicolumn{1}{|c|}{ Low time costs } & Low time costs \\
\hline DVRP & Low time costs & \multicolumn{1}{|c|}{ High time costs } & High time costs \\
\hline Causes & $\begin{array}{l}\text { The computational complexity } \\
\text { of LSRP is higher than DVRP, } \\
\text { exceeding 1.52 times of } \\
\text { convergence time in the } \\
\text { simulation environment (seen } \\
\text { in Figure 3). }\end{array}$ & $\begin{array}{l}\text { The routing of LSRP doesn't change after the } \\
\text { routing is convergent, and except for LSA } \\
\text { information interaction, other routing information } \\
\text { is not sent. DVRP should send the routing table to } \\
\text { the neighbor routings in the period time, and this } \\
\text { action occupies CPU and bandwidth time (seen in } \\
\text { Figure 2 and Figure 4). }\end{array}$ & $\begin{array}{l}\text { LSRP sends less } \\
\text { information, occupies } \\
\text { fits for large-scale } \\
\text { network. }\end{array}$ \\
\hline
\end{tabular}




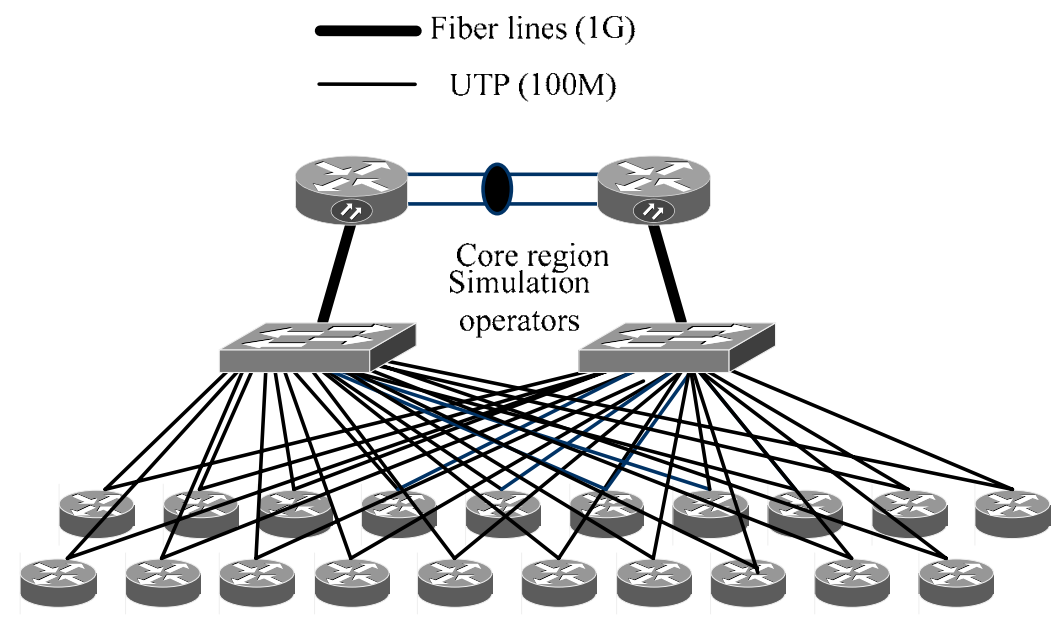

Service network

Figure 1. Simulation Network Topology

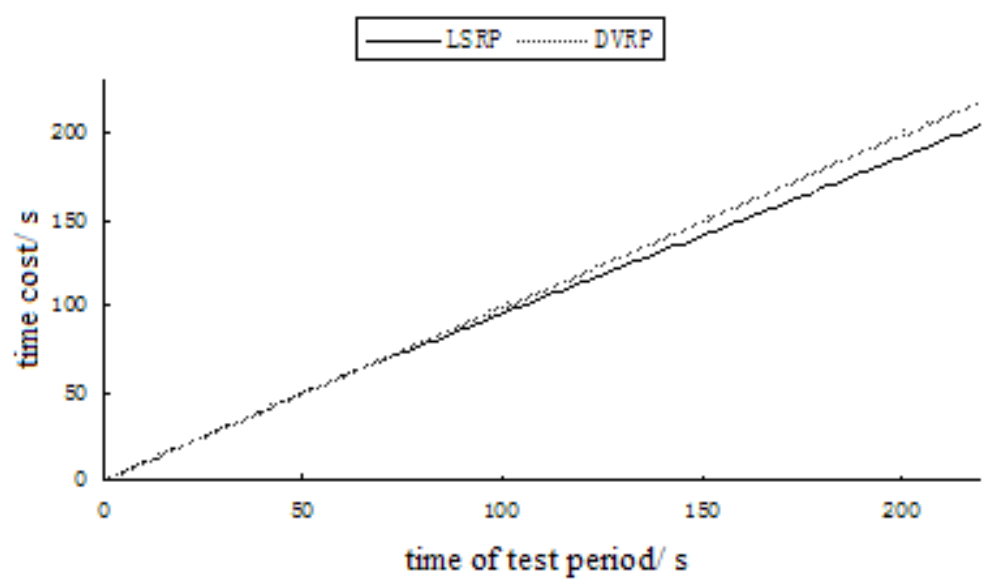

Figure 2. Comparison of Time Costs of LSRP and DVRP

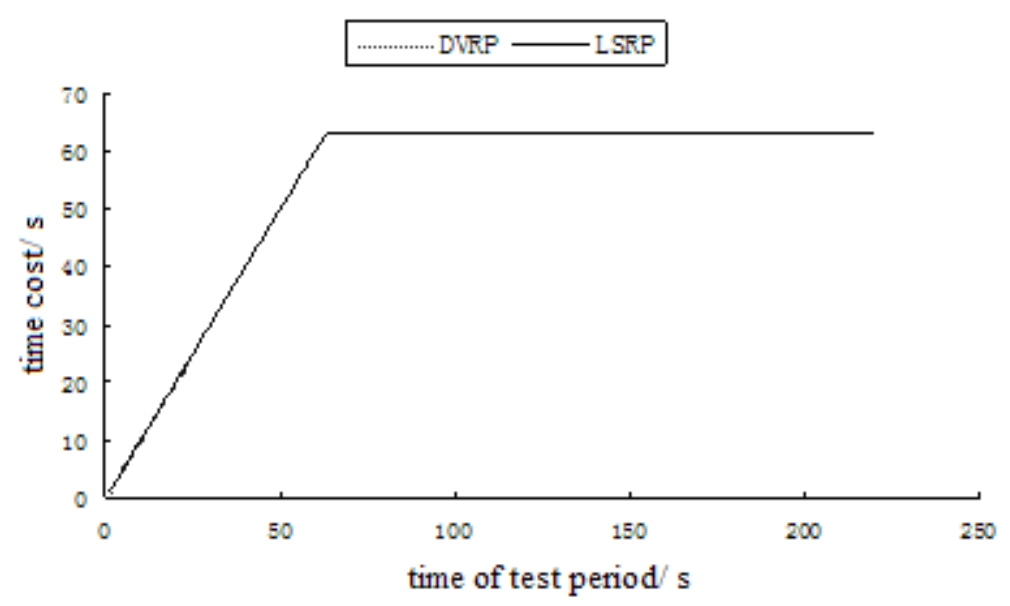

Figure 3. Comparison of Convergence Time Costs of LSRP and DVRP 


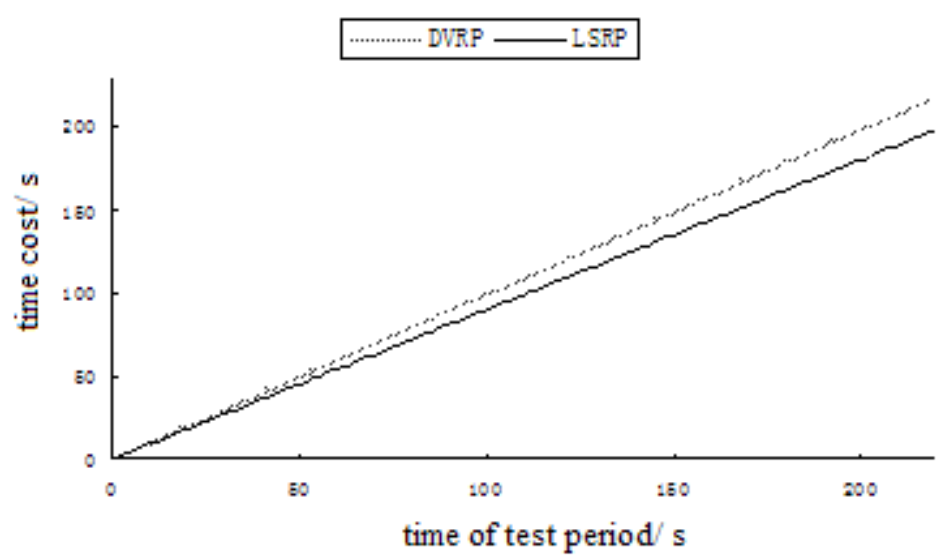

Figure 4. Comparison of Bandwidth Time Costs of LSRP and DVRP

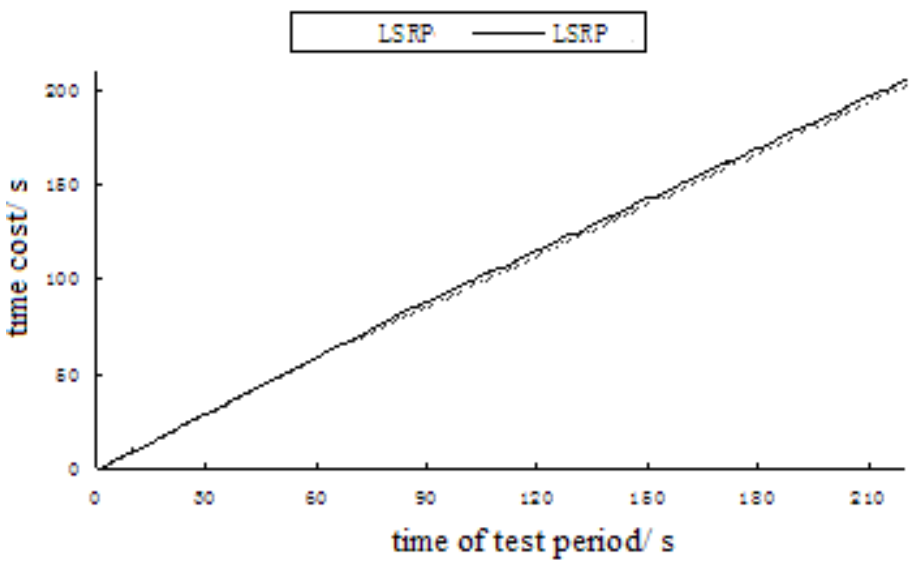

Figure 5. Comparison of Simulation and Reality Comprehensive Time Costs of LSRP

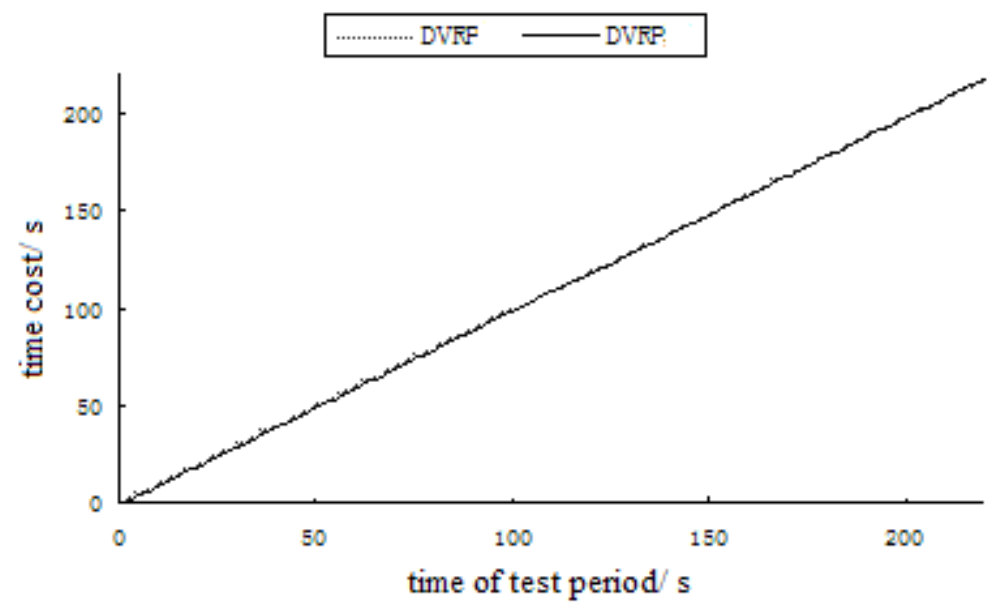

Figure 6. Comparison of Simulation and Reality Comprehensive Time Costs of DVRP 